\title{
Use of Polypropylene Strips for Reinforcement of the Cruroplasty in Laparoscopic Paraesophageal Hernia Repair: A Retrospective Cohort Study
}

\author{
L. Matthijs Van Den Dop ${ }^{a}$ Gijs H.J. De Smet ${ }^{a} \quad$ Aziz Mamound $^{b} \quad$ Johan Lange $^{\mathrm{a}, \mathrm{c}}$ \\ Bas P.L. Wijnhoven ${ }^{a}$ Willem Hueting ${ }^{b}$
}

aDepartment of Surgery, Erasmus University Medical Centre, Rotterdam, The Netherlands; ${ }^{b}$ Department of Surgery, Alrijne Ziekenhuis, Leiderdop, The Netherlands; 'Department of Surgery, IJsselland Ziekenhuis, Capelle aan den IJssel, The Netherlands

\section{Keywords}

Laparoscopic correction · Paraesophageal hernia · Mesh ·

Polypropylene strips · Recurrence

\begin{abstract}
Introduction: Laparoscopic paraesophageal hernia repair is an effective treatment for symptomatic paraesophageal hernias. To reduce recurrence rates, the use of prosthetics for the crural repair has been suggested. Mesh-related complications are rare but known to be disastrous. To address another form of crural repair, polypropylene strips are suggested. This study aimed to assess peri- and postoperative complications of reinforcement of cruroplasty with polypropylene strips. Methods: From 2013 to 2020, patients with a primary or recurrent type 2, 3, or 4 paraesophageal hernia that underwent cruroplasty with polypropylene strips were retrospectively reviewed. Intra- and postoperative complications were graded according to the Clavien-Dindo classification. The incidence of symptomatic recurrent hiatal hernia (CT or endoscopy proven) and hospital stay were assessed. Results: One hundred fifty-eight patients were included. Mean age was 65 years (standard deviation 10.4), and 119 patients were female (75.3\%). Almost $50 \%$ of surgeries took place between 2018 and 2020. Median follow-up was 7 months (in-
\end{abstract}

terquartile range 17.5). Mean operation time in the primary hernia group was 159 min (standard deviation 39.0), and length of stay was 4.4 days. In 3/158 patients (2.0\%), intraoperative complications occurred. Two patients developed a grade 4 and seven patients a grade 3 postoperative complication. No mortality was recorded. Twelve recurrences (8.2\%) were detected in the primary hernia group and one $(9.1 \%)$ in the recurrent hernia group. Conclusion: There were no mesh-related complications seen and symptomatic recurrence rate was low, but longer follow-up is needed.

(c) 2021 The Author(s).

Published by S. Karger AG, Basel

\section{Introduction}

Laparoscopic repair of a paraesophageal hernia $(\mathrm{PEH})$ is performed to relieve disabling symptoms and to prevent complications. The majority of patients have satisfactory outcomes [1-4]. However, recurrence rates after 1 year in patients who underwent laparoscopic $\mathrm{PEH}$ repair are reported to be $19.4 \%$ [5].

To reduce the recurrence rates after laparoscopic $\mathrm{PEH}$ repair, the use of prosthetics has been studied. Oelschlager et al. [6] evaluated the use of an absorbable mesh for re- karger@karger.com www.karger.com/dsu

Karger $\stackrel{\text { ' }}{5}$

BOPEN ACCESS
(C) 2021 The Author(s)

Published by S. Karger AG, Basel

This is an Open Access article licensed under the Creative Commons Attribution-NonCommercial-4.0 International License (CC BY-NC) (http://www.karger.com/Services/OpenAccessLicense), applicable to the online version of the article only. Usage and distribution for commercial purposes requires written permission.
Correspondence to:

L. Matthijs Van den Dop, l.vandendop@erasmusmc.nl 
inforcement of the posterior hiatal closure in a randomized clinical trial. The recurrence rates at 5 -year follow-up were $54 \%$ in the absorbable mesh group and 59\% in the primary suture group. No mesh-related complications were reported. Recently, Oor et al. [5] also published a randomized clinical trial in which non-absorbable mesh closure and suture closure of the hiatal defect were compared. After 1 year, no significant differences in the proportion of recurrent hiatal hernias were found (19.4 vs. $11.4 \%)$, and both groups had similar dysphagia and satisfaction scores. No mesh-related complications occurred. Other studies reported similar outcomes for symptomatic recurrence rates and complications $[7,8]$.

Although studies do not support the routine use of meshes for PEH defect repair, some surgeons feel that mesh is indicated for large PEH repair. However, complications associated with the use of mesh are erosion in the oesophagus or stomach, migration, and oesophageal stenosis due to fibrosis [9]. These complications may be explained by the dynamics of the crural pillars, which induces friction between the mesh and other viscera [9].

To minimize the risk of these mesh related complications, the use of small polypropylene strips to reinforce the cruroplasty has been suggested [10]. The concept of this technique is to induce mesh-related fibrosis and strengthening of the cruroplasty to reduce recurrences, while preventing friction between the prosthetics and viscera. The purpose of the present study was to evaluate perioperative complications and to assess recurrence rate of the polypropylene strip-reinforced cruroplasty (PSRC) technique.

\section{Materials and Methods}

This retrospective observational study was conducted according to the STROBE and STROCSS statements $[11,12]$.

\section{Study Design}

All patients who underwent primary or recurrent laparoscopic hiatal hernia repair with PSRC in a non-academic referral (the Alrijne) hospital in the Netherlands between 2013 and 2020 were identified from the hospital records. The Local Ethics Committee of the Alrijne hospital approved the study protocol. Study data analysed were stored on the server of the Alrijne hospital.

Patients with a primary or recurrent symptomatic type 2, 3, or $4 \mathrm{PEH}$ were included, confirmed by preoperative endoscopy and barium swallow test and/or CT. Patients were excluded if they had an American Society of Anaesthesiologists (ASA) classification of $>3$, did not wish to receive PSRC, received other strips than polypropylene strips, were pregnant, had an emergency procedure, or were younger than 18 years of age. The type 1 hiatal hernias were excluded considering the different epidemiology, pathophysiology, and relatively small hernia sizes.

Paraesophageal Hernia Repair with Strips

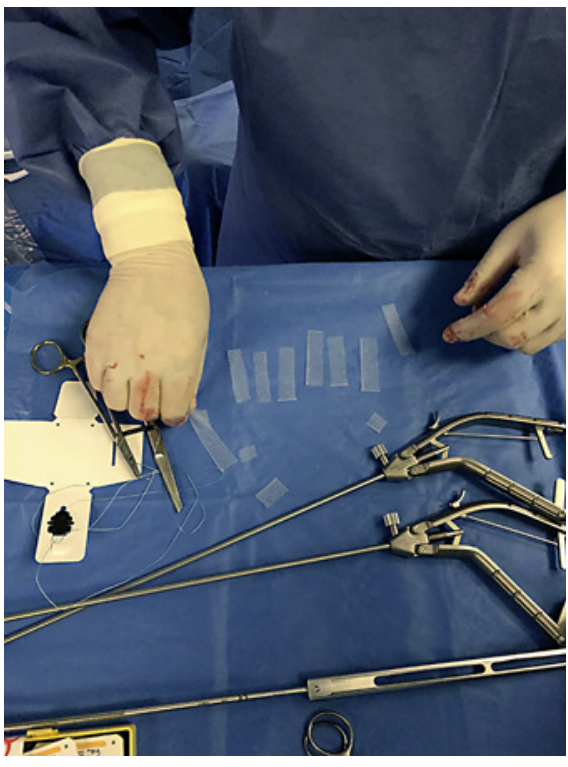

Fig. 1. Polypropylene strips created from a standard polypropylene mesh (Prolene).

Patients were first seen at the outpatient clinic by a surgeon who performed all surgical procedures (W.H.) and a gastroenterologist. Gastroscopy, barium swallow test, and/or a CT were performed. All patients were discussed at a multidisciplinary team meeting with the surgeon, a dedicated gastroenterologist, and a radiologist. Oesophageal manometry was performed if there was suspicion of motility disorders after multidisciplinary consultation. The use of the polypropylene strips was part of the standard care, and patients were informed about the use of prosthetic strips. Recurrent $\mathrm{PEH}$ was only included when the first operation was performed without PSRC before 2013 or when patients were referred from another hospital.

The medical records of each patient were retrospectively reviewed to assess patient characteristics (sex, age at surgery, BMI, and ASA classification). Hernia characteristics (type of hiatal hernia), surgical characteristics (operation time, length of stay [LOS], type of mesh, and number of strips used), intraoperative complications, and postoperative outcomes (recurrence, dysphagia, and return of proton pump inhibitor [PPI] use) were assessed during follow-up appointments.

\section{Surgical Procedure}

All procedures were performed using a standardized laparoscopic technique under general anaesthesia. Antibiotic prophylaxis was given 30 minutes before skin incision (cefazolin $1 \mathrm{~g}$ and metronidazole $500 \mathrm{mg}$ ). The first step was dissection and reposition of the hernia sac, stomach, and, when present, other abdominal organs. Next, the hiatus was fully exposed and the distal oesophagus and gastric fundus fully mobilized for further manipulation. The distal oesophagus was mobilized in the chest in order to bring down the gastro-oesophageal junction without tension, preventing tension and traction on the oesophagus. Division of the short gastric vessels was always performed. After mobilization, the crural repair was performed. 

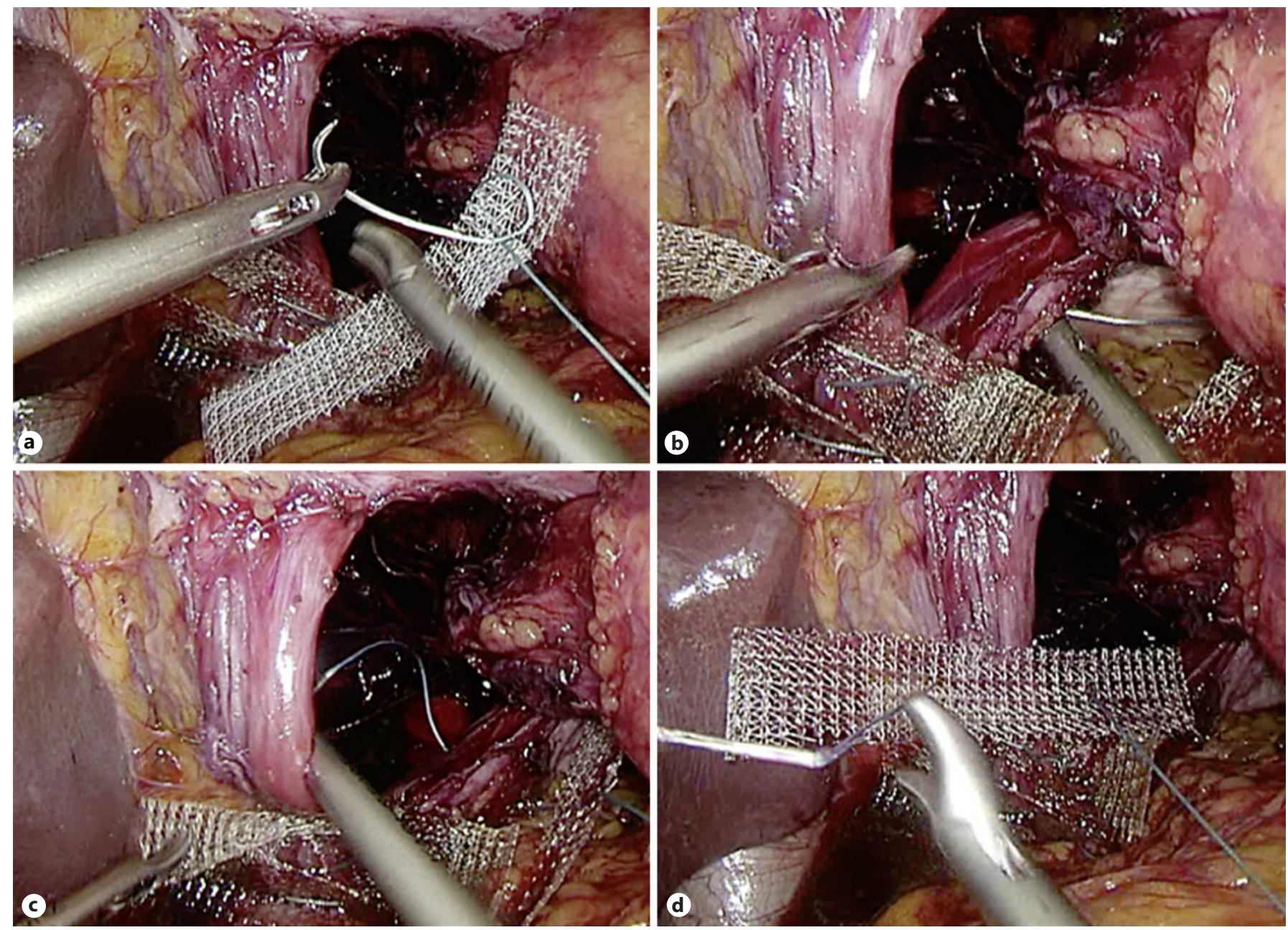

Fig. 2. Crural repair using PSRC. The suture is brought through the strip (a), suture is brought through the left crural pillar (b), suture is brought through the right pillar (c), and finally the suture is brought back through the strip (d). PSRC, polypropylene strip-reinforced cruroplasty.

\section{Crural Repair}

Polypropylene strips of $3 \mathrm{~cm}$ in length and $1 \mathrm{~cm}$ in width were cut from a standard polypropylene mesh (Prolene; Ethicon Inc., Somerville, NJ, USA) or a polypropylene mesh with coating to prevent adhesion formation (C.R. Bard, Murray Hill, NJ, USA) (Fig. 1). The posterior crural repair was performed with woven non-absorbable sutures (Ethibond; Ethicon Inc., Somerville, NJ, USA). First, the suture was brought through the strip (Fig. 2a) and then through the left crural pillar (Fig. 2b), the right pillar (Fig. 2c), and back through the strip again (Fig. 2d). A knot pusher was used to get tactile feedback of the tension of the reinforcement, making sure the strips are stitched tension free to the crural pillars. The number of strips used was determined intraoperatively by stripby-strip approximation. This enabled the surgeon to perform the repair for all 3 types of $\mathrm{PEH}$. The most ventral sutures were tied without using a strip to avoid direct contact with the oesophagus. Normally, one suture was used for the ventral side. If the ventral hiatus was deemed too wide still, another suture was used to ap- proximate the defect. In addition, a $270^{\circ}$ posterior Toupet fundoplication was performed to avoid reflux and to reinforce the posterior crural repair.

The fundoplication was created by placing two sutures between the wrap and suturing this against the strips and the crural pillars, achieving a firm attachment (Fig. 3a). The middle section of the fundoplication was sutured to the right side of the oesophagus (Fig. 3b), and the left side of the fundoplication stitches was stitched to the left side of the oesophagus, with exception of the most cranial suture, which was placed to the left side of the diaphragm (Fig. 3c). By fixating the fundoplication on to the polypropylene strips, friction was avoided between the polypropylene strips and the oesophagus (Fig. 3d).

\section{Outcomes}

Because of the difference in patient outcomes and surgical risks between the $\mathrm{PEH}$ repair and recurrent $\mathrm{PEH}$ repair, these two groups were analysed separately. The primary outcome was 

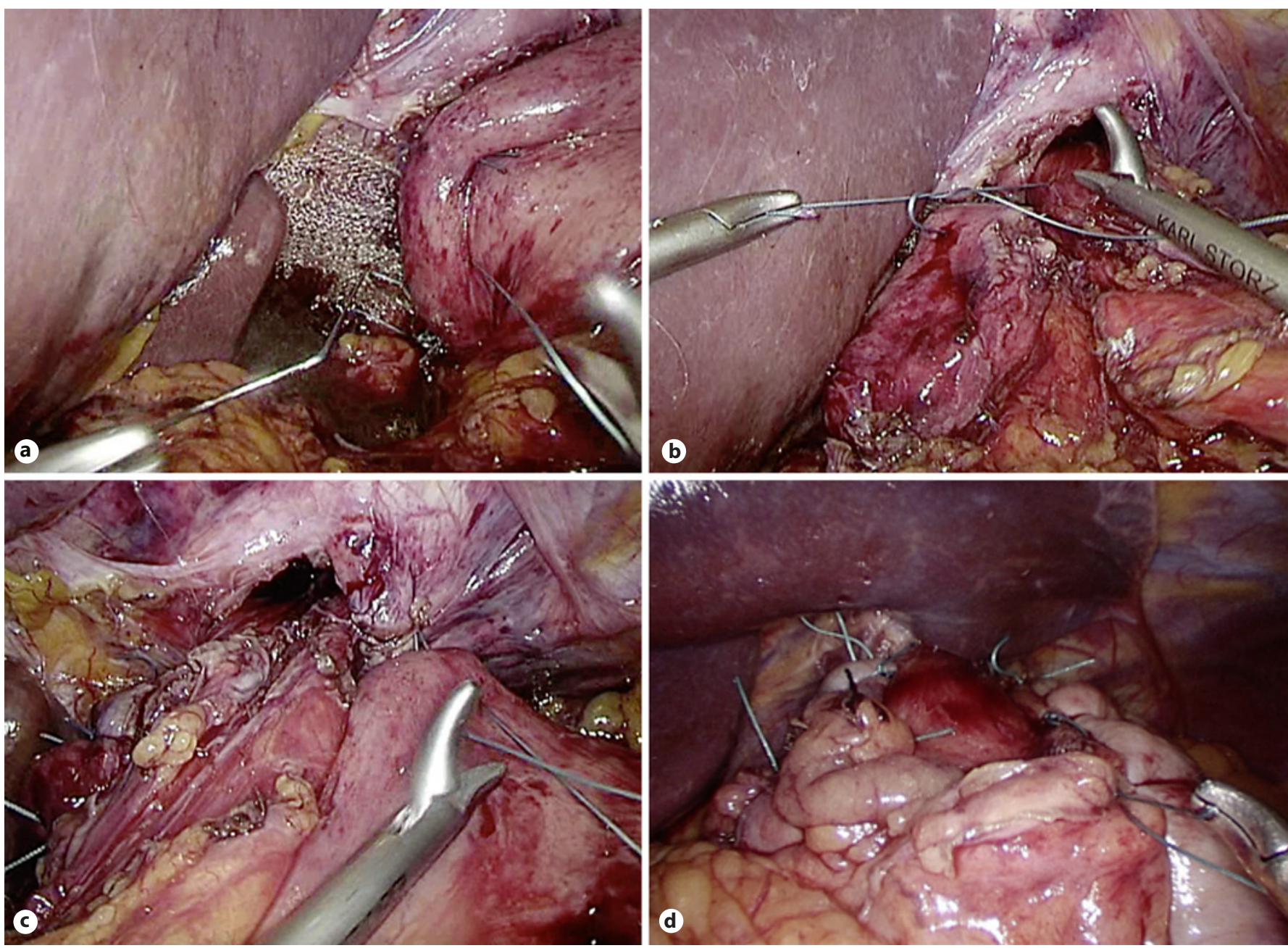

Fig. 3. Fundoplication as a natural barrier for the oesophagus. The caudal right side of the fundoplication being sutured to the crural pillars and the strips (a), the middle section of the fundoplication was sutured to the right side of the oesophagus (b), the most cranial suture of the left side of the fundoplication was sutured to the left side of the diaphragm (c), and fundoplication acting as a natural barrier (d).

perioperative complications categorized according to the Clavien-Dindo classification for surgical complications [13]. When the intraoperative and postoperative complications were comparable to other studies reported in the literature and not related to the use of mesh, the PSRC technique was considered a safe procedure.

Secondary outcomes were LOS (in days), operation time (in minutes), persistent dysphagia (defined as dysphagia for solids lasting $>1$ year), return to PPI use, and recurrences. A recurrence was defined as a symptomatic herniation of the stomach or other abdominal organs in the mediastinum or the presence of a new $\mathrm{PEH}$ as diagnosed by gastroscopy or a radiological modality (i.e., barium swallow test or CT). Symptoms or signs that led to radiological or gastroscopic investigation were no resolution of preoperative symptoms, progressive retrosternal pain, dysphagia, reflux, and return of symptoms after heavy coughing.

Paraesophageal Hernia Repair with Strips
Follow-Up

Patients were seen two and six weeks after the operation. No routine barium swallow test, gastroscopy, $\mathrm{pH}$ monitoring, or manometry was done. When patients did not develop new symptoms and were satisfied, they were discharged from further follow-up.

Patients were instructed to contact the hospital if new symptoms emerged. A barium swallow test was performed in patients who experienced lasting symptoms after the 6-week follow-up appointment. If this barium swallow test showed a normal anatomy, another appointment was made after three months. In case of persistent symptoms after three months, the patient was referred to a multidisciplinary consultation team, and a gastroscopy and/or CT scan was performed. If no clear pathology was found, an additional oesophageal motility and $\mathrm{pH}$ monitoring investigation was performed. 


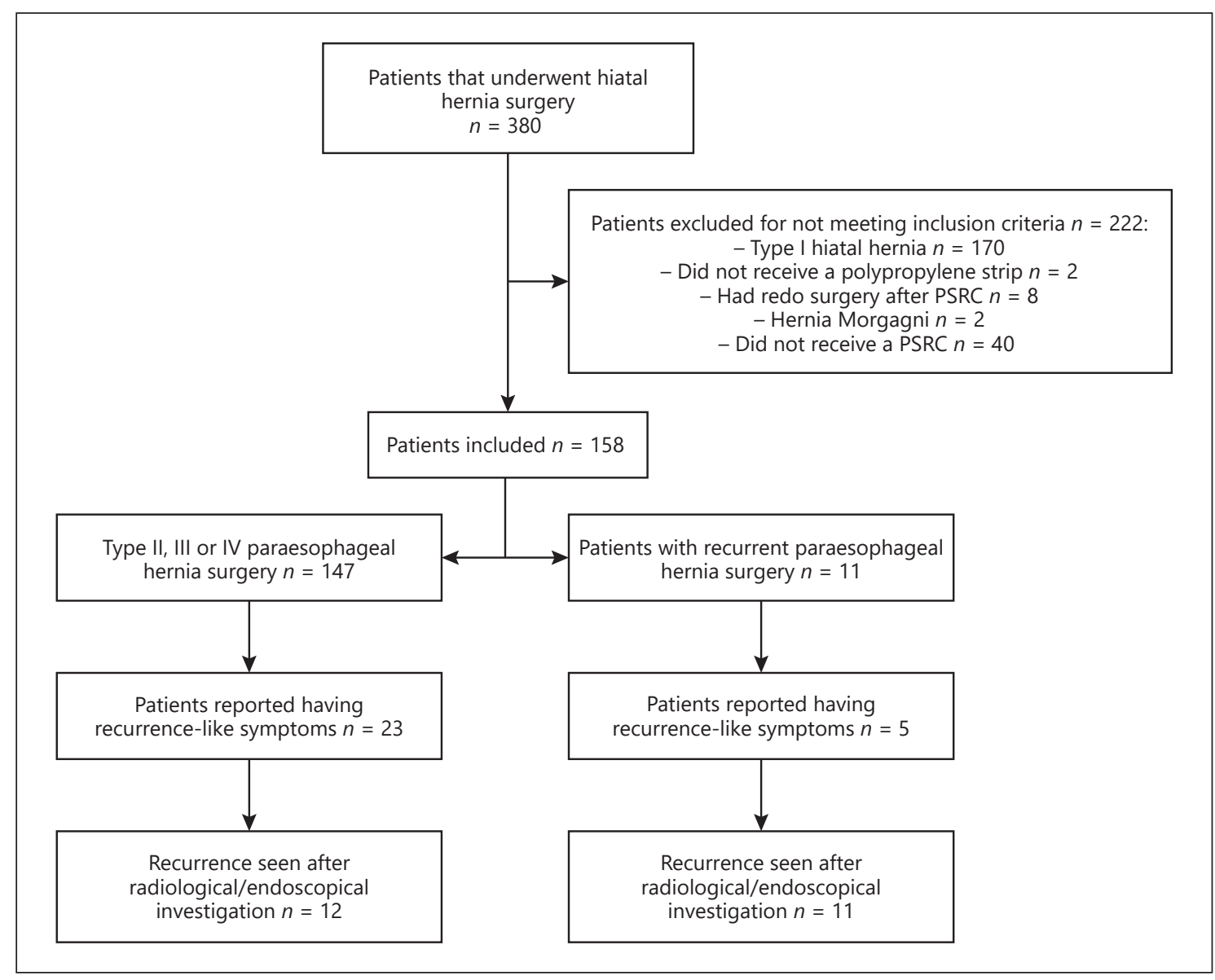

Fig. 4. Flowchart representing patient outcome. PSRC, polypropylene strip-reinforced cruroplasty.

\section{Statistical Analysis}

Statistical analysis was performed with SPSS software version 26 (IBM SPSS Statistics for Mac version 26; IBM Corporation, Armonk, NY, USA). Continuous variables are presented as median and interquartile range or median. Discrete variables are presented as absolute numbers and percentages.

\section{Results}

A total of 380 patients underwent laparoscopic hiatal surgery between 2013 and 2020. One hundred seventy patients were excluded because they had a type 1 hiatal hernia, eight patients who had a redo after PSRC, and two patients had a Morgagni-type hernia. Another 40 patients were excluded because they did not receive PSRC, and two patients were excluded because they received bio-absorbable strips, leaving 158 patients for review (Fig. 4). Patient characteristics are shown in Table 1. The most prevalent type of paraesophageal hernia was a type 4 her- nia in 79 patients (50.0\%). Median follow-up was seven months with an interquartile range of 17.5 months.

\section{Surgical Characteristics}

Almost 50\% of the surgeries took place at the start of 2018. Mean operation time was $159 \mathrm{~min}$ (SD $39.0 \mathrm{~min}$ ), and mean LOS was 4.4 days (SD 2.9 days), shown in Table 2. In most procedures, 3 strips were used for reinforcing the cruroplasty (40.8\%), and a Prolene mesh was used most often to construct the strips $(96.6 \%)$. Two conversions (1.4\%) to an open procedure occurred: one conversion because of a lesion to the oesophagus and one conversion because of severe adhesions in the upper abdomen.

\section{Perioperative Complications}

Three intraoperative complications occurred during the PSRC surgeries, including a lesion of the oesophagus which required a conversion to an open procedure and 
Table 1. Baseline characteristics for patients with PSRC

\begin{tabular}{lc}
\hline PSRC & $N=158(\%)$ \\
\hline Sex & \\
Male & $39(24.7)$ \\
Female & $119(75.3)$ \\
Age, years & $65(10.4)$ \\
BMI, kg/m 3 & $29(4.3)$ \\
ASA classification & \\
1 & $9(5.7)$ \\
2 & $104(65.8)$ \\
3 & $45(28.5)$ \\
Type of paraesophageal hernia & $11(7.0)$ \\
Type 2 & $57(36.1)$ \\
Type 3 & $79(50.0)$ \\
Type 4 & $11(7.0)$ \\
Redo of paraesophageal hernia & 4 \\
Type 2 & 4 \\
Type 3 & 3 \\
Type 4 & $7(17.5)$ \\
Follow-up, months & \\
\hline
\end{tabular}

Continuous variables are presented as mean and SD, except for follow-up, which is presented as median with IQR. Discrete variables are presented as absolute number and percentage. ASA, American Society of Anaesthesiologist; PSRC, polypropylene strip-reinforced cruroplasty.

primary closure (Table 3). All patients recovered without further sequelae.

There were three patients with a grade 4 complication and seven patients with a grade 3 complication (Table 3 ). All patients fully recovered. The grade 1 and grade $2 \mathrm{com}$ plications included antibiotic and heparin treatments, requirement of additional analgesia, and obstipation.

\section{Postoperative Symptoms}

The postoperative symptoms, recurrence rate, return to PPI use, and dysphagia are shown in Table 4. Twentyeight patients reported recurrence-like symptoms (Fig. 4). These patients were seen at the outpatient clinic and underwent radiological and/or endoscopic investigation. Twenty-five (15.8\%) of the patients reported PPI use after the operation, and four patients reported having persistent dysphagia (2.5\%).

\section{Symptomatic Recurrences}

Thirteen patients developed a symptomatic recurrence $(8.2 \%)$. Seven recurrences occurred in the PEH type 4 group (4.8\%), 5 in the type 3 group (3.3\%), and $1(0.6 \%)$ in the redo group (type 4). Symptoms leading to investi-
Table 2. Surgical characteristics for patients with PSRC

\begin{tabular}{|c|c|}
\hline PSRC & $N=158(\%)$ \\
\hline Type 2,3 , or 4 paraesophageal hernia & $N=147$ \\
\hline Operation time, $\min$ & $159(39.0)$ \\
\hline \multicolumn{2}{|l|}{ Strips used for cruroplasty, $n$} \\
\hline 1 strip & $22(15.0)$ \\
\hline 2 strips & $34(23.1)$ \\
\hline 3 strips & $60(40.8)$ \\
\hline 4 strips & $26(17.7)$ \\
\hline 5 strips & $4(2.7)$ \\
\hline \multicolumn{2}{|l|}{ Type of mesh for strips used } \\
\hline Prolene $\mathrm{a}^{\mathrm{a}}$ & $142(96.6)$ \\
\hline Ventralight $^{\mathrm{b}}$ & $2(1.4)$ \\
\hline Sepramesh ${ }^{\mathrm{b}}$ & $3(2.0)$ \\
\hline Conversion to open procedure & $2(1.4)$ \\
\hline LOS, days & $4.4(2.9)$ \\
\hline Redo of paraesophageal hernia recurrence & $N=11$ \\
\hline Operation time, $\min$ & $159(21.8)$ \\
\hline \multicolumn{2}{|l|}{ Strips used for cruroplasty, $n$} \\
\hline 1 strip & $4(36.7)$ \\
\hline 2 strips & $7(63.3)$ \\
\hline \multicolumn{2}{|l|}{ Type of mesh for strips used } \\
\hline Prolene & $10(90.9)$ \\
\hline Marlex & $1(9.1)$ \\
\hline LOS, days & $3.7(2.6)$ \\
\hline
\end{tabular}

Continuous variables are presented as mean and SD. Discrete variables are presented as absolute number and percentage. PSRC, polypropylene strip-reinforced cruroplasty; LOS, length of stay.

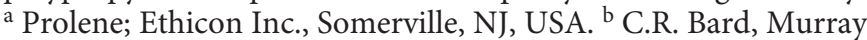
Hill, NJ, USA.

gation consisted of epigastric discomfort, reflux complaints, dyspnoea, and dysphagia.

Four patients received four strips, another four patients three strips, three patients two strips, one patient five strips, and one patient one strip. Six patients were diagnosed by a CT scan, another six with a barium swallow test, and one patient during gastroscopy. Mean time to discovery of the recurrence was 22.3 months.

\section{Discussion}

This retrospective cohort study evaluated perioperative complications and short-term recurrence of the PSRC technique in PEH surgery and showed a low rate of intraoperative complications (1.9\%), as well as low symptomatic recurrence rate $(8.2 \%)$. Intraoperative complications occurring during primary $\mathrm{PEH}$ mesh-reinforced cruroplasty have been reported to be $7.3 \%$ and $4.8 \%$ in the 
Table 3. Perioperative complications after PSRC

\begin{tabular}{|c|c|}
\hline & $N=147$ \\
\hline Type 2, 3, or 4 with PSRC & $N=158(\%)$ \\
\hline Intraoperative complications & $2(1.3)$ \\
\hline Bleeding of preperitoneal lipoma & 1 \\
\hline Lesion to the oesophagus & 1 \\
\hline Postoperative complications ${ }^{\mathrm{a}}$ & $45(30.6)$ \\
\hline Grade 1 & $23(15.6)$ \\
\hline Grade 2 & $13(8.8)$ \\
\hline Pneumonia requiring antibiotics & 9 \\
\hline Pulmonary embolism & 2 \\
\hline Splenic emboli & 1 \\
\hline Delirium & 1 \\
\hline Grade $3 \mathrm{a}$ & $5(3.4)$ \\
\hline Drainage of subphrenic abscess & 1 \\
\hline Respiratory acidosis & 1 \\
\hline Haematemesis & 1 \\
\hline Nasogastric tube feeding due to severe dysphagia & 2 \\
\hline Grade $3 b$ & $2(1.4)$ \\
\hline Rupture of diaphragm & 1 \\
\hline Respiratory insufficiency & 1 \\
\hline Grade 4 & $2(1.4)$ \\
\hline Acute renal failure requiring ICU monitoring & 1 \\
\hline Systematic inflammatory response syndrome & 1 \\
\hline Redo of paraesophageal hernia with PSRC & $N=11$ \\
\hline Intraoperative complications & $1(15.4)$ \\
\hline Subscapular bleeding of the liver & $1(9.1)$ \\
\hline Postoperative complications ${ }^{\mathrm{a}}$ & $3(27.3)$ \\
\hline Grade 1 & $1(9.1)$ \\
\hline Grade 2 & $2(18.2)$ \\
\hline Pneumonia requiring antibiotics & 1 \\
\hline Blood transfusion after subscapular bleeding of the liver & 1 \\
\hline
\end{tabular}

Continuous variables are presented as mean and SD. Discrete variables are presented as absolute number and percentage. PSRC, polypropylene strip-reinforced cruroplasty. ${ }^{a}$ Graded with the Clavien-Dindo classification of postoperative complications (4). study by Watson et al. [8] and $5.6 \%$ in the study by Oor et al. [5]. The intraoperative complication rate of primary suture cruroplasty was reported to be $8.3 \%, 7.0 \%$, and $18 \%[5,6,8]$. The intraoperative complication rate in the primary PEH group was 2/147 (1.3\%), and the complications were not related to the PSRC technique itself. This could indicate that the PSRC technique used in this study could be regarded as a safe and feasible operative procedure.

Postoperative complications occurring after prosthetic reinforced cruroplasty are reported to be $11.7 \%$ in a study which analysed outcomes from a national database with almost 9,000 patients [14]; however, type of hiatal hernia was not reported. Other similar studies reported incidences of $10-27 \%[6,8]$. Our study included minor complications including patients who needed additional analgesia or had problems with obstipation. The incidence of a grade 4 complication in our study was low $(1.4 \%)$.

The use of polypropylene strips to reinforce the cruroplasty has been investigated before by Granderath et al. [10]. They reported no intraoperative complications, no symptomatic and radiological recurrences, no mesh-related complications, or other complications in twelve patients six months after surgery. The use of meshes in PEH repair is still controversial, as severe mesh-related complications have been reported (e.g., mesh erosion, infection, and oesophageal stenosis requiring re-interventions) [9]. It is hypothesized that migration of the mesh at the hiatus may occur due to intermittent (coughing and straining) or ongoing (breathing) diaphragmatic contractions and relaxations [9]. Therefore, routine mesh fix- 
Table 4. Postoperative symptoms after PSRC

\begin{tabular}{lc}
\hline & $N=147$ \\
\hline Type 2, 3, or 4 with PSRC & $N=158(\%)$ \\
Recurrence & $12(8.1)$ \\
Type 2 & 0 \\
Type 3 & $5(3.4)$ \\
Type 4 & $7(4.8)$ \\
Return to PPI use & $21(14.3)$ \\
Persistent dysphagia & $3(2.0)$ \\
\hline Redo of paraesophageal hernia with PSRC & $N=11$ \\
Recurrence & $1(9.1)$ \\
Type 4 & $1(9.1)$ \\
Return to PPI use & $4(36.4)$ \\
Persistent dysphagia & $1(9.1)$ \\
\hline
\end{tabular}

Continuous variables are presented as mean and SD. Discrete variables are presented as absolute number and percentage. PPI, proton pump inhibitor; PSRC, polypropylene strip-reinforced cruroplasty.

ation is not advised to cover the crural defect. The concept of using polypropylene strips for crural reinforcement done in the present study is as follows: first, the strips were small and placed dorsally on the crural pillars. Second, the purpose of placing the strip was to evenly spread tension on the stitched crural pillars. Third, polypropylene causes fibrosis, and this may strengthen the cruroplasty. Fourth, the strips secured a tight connection of the fundoplication with the pillars. The technique that was used in this study prevents erosion of the strips to the gastric wall and oesophagus during movements or traumatic events. Furthermore, peritoneal overgrow on the right crural pillar is seen in patients operated for a recurrence and may prevent contact from the strip with other abdominal viscera. In this study, no mesh-related complications (i.e., oesophageal stenosis, mesh infection, or migration) were observed.

The cost of a Prolene (Ethicon Inc., Somerville, NJ, USA) mesh was EUR 15, in this hospital. The bio-resorbable meshes used in some studies may have a lower risk for mesh-related complications; however, these are expensive. The cost of a Phasix (C.R. Bard, Murray Hill, NJ, USA) mesh was around EUR 500. Furthermore, the longterm follow-up recurrence rates seen in the study by Oelschlager et al. [6] are almost comparable to those with primary suture cruroplasty.

In a meta-analysis of randomized controlled trials of Memon et al. [7], a total of 215 patients with prosthetic reinforced cruroplasty in large hiatal hernias were anal-

Paraesophageal Hernia Repair with Strips ysed, and 35 recurrences were objectified (16.3\%). One hundred eighty-two patients that underwent primary suture repair were also analysed, and 50 recurrences (27.4\%) were found. In another meta-analysis of Campos et al. [15], no evidence was found that routine mesh reinforcement in laparoscopic repair of giant $\mathrm{PEH}$ decreases recurrences, compared to suture repair $(p=0.12)$. Finally, the study by Watson et al. [16] also suggests that mesh reinforcement does not significantly reduce recurrence rates. The short-term recurrence rate found in the present study is low (8.2\%). This could be explained due to the fact that no standard postoperative radiological follow-up was scheduled and indicates that some paraesophageal recurrences were asymptomatic or mildly symptomatic not seeking medical attention. However, patients were clearly instructed to see us at the outpatient clinic in case of recurrent symptoms.

One of the complications after laparoscopic PEH repair and especially with use of mesh is persistent dysphagia. This may occur after the use of non-absorbable prosthetics due to fibrosis around the oesophagus [9]. The contact of meshes with the oesophagus should therefore be avoided [9]. However, since the hiatus is a dynamic anatomical structure, complete prevention of mesh contacting the oesophagus is difficult $[9,17]$. The short-term incidence of postoperative dysphagia in non-resorbable prosthetic reinforced cruroplasty is reported to be 17.5$23.5 \%[5,8,18]$. In a study by Dallemagne et al. [19], the occurrence of persistent dysphagia was approximately in $3 \%$ of cases 5 years after surgical treatment of gastro-oesophageal reflux disease by laparoscopy. They did not use prosthetics to reinforce the cruroplasty. The incidence of persistent dysphagia in this study is reported to be $2.0 \%$, which was lower, and with the use of prosthetics. However, no use was made of validated dysphagia questionnaires.

This study has some limitations. No pre- or postoperative validated questionnaires were used to assess the pre- and postoperative symptoms of the patients. Another limitation was the lack of standardized follow-up appointments, including barium swallow studies. Since $>50 \%$ of the surgeries took place between the end of 2017 and 2020, follow-up duration was limited. This may have led to an underestimation of the recurrence rate. However, one of the reasons the recurrence rate in this study is low could be the multidisciplinary consultation meeting. Comorbidities, preoperative tests, and conservative pharmaceutical treatment were discussed before making the decision to perform surgery. By accomplishing these consultations, a patient population was formed that 
would benefit the most out of this surgery and therefore aimed to minimalize the postoperative complaints and complications.

\section{Conclusion}

The findings from this study show a low incidence of perioperative complications after PEH repair with PSRC. Complications were not related to the PSRC technique itself. The short-term recurrence rate was low, as was dysphagia after surgery, and no mesh-related complications were observed. Longer follow-up is needed to affirm low recurrence rates. The use of small polypropylene strips to reinforce the cruroplasty is a promising concept and a safe and feasible technique.

\section{Statement of Ethics}

All authors comply that this research was conducted ethically in accordance with the World Medical Association Declaration of Helsinki. The local ethics committee reviewed and approved the study protocol (Reference No. NWMO 20-07). Furthermore, the Local Ethics Committee of the Alrijne hospital stated that no informed consent of individual patients was required for the retrospective data collection for this study. All patients under treatment in the Alrijne hospital automatically give informed consent to use their anonymized medical data for research purposes, if standard care is given.

\section{Conflict of Interest Statement}

L.M. Van den Dop, MD, G.H.J. De Smet, MD, A. Mamound, $\mathrm{MD}$, J. Lange, $\mathrm{MD}$, PhD, B.P.L. Wijnhoven, $\mathrm{MD}, \mathrm{PhD}$, and W.E. Hueting, $\mathrm{MD}, \mathrm{PhD}$, have no conflicts of interest of financial ties to disclose.

\section{Funding Sources}

No financial support was received in support of this manuscript.

\section{Author Contributions}

Van den Dop was involved in data assembly and writing of the manuscript. De Smet wrote the manuscript. Mamound edited and corrected the manuscript. Lange edited and corrected the manuscript. Wijnhoven edited and corrected the manuscript. Hueting performed surgeries, editing, and correcting of the manuscript.

\section{Data Availability Statement}

Due to privacy and ethical concerns, the data can not be made publically available.

\section{References}

1 Mattar SG, Bowers SP, Galloway KD, Hunter JG, Smith CD. Long-term outcome of laparoscopic repair of paraesophageal hernia. Surg Endosc. 2002;16:745-9.

2 Luketich JD, Raja S, Fernando HC, Campbell W, Christie NA, Buenaventura PO, et al. Laparoscopic repair of giant paraesophageal hernia: 100 consecutive cases. Ann Surg. 2000; 232:608-18

3 Karmali S, McFadden S, Mitchell P, Graham A, Debru E, Gelfand G, et al. Primary laparoscopic and open repair of paraesophageal hernias: a comparison of short-term outcomes. Dis Esophagus. 2008;21:63-8.

4 Dallemagne B, Kohnen L, Perretta S, Weerts J, Markiewicz S, Jehaes C. Laparoscopic repair of paraesophageal hernia. Long-term followup reveals good clinical outcome despite high radiological recurrence rate. Ann Surg. 2011; 253:291-6.

5 Oor JE, Roks DJ, Koetje JH, Broeders JA, van Westreenen HL, Nieuwenhuijs VB, et al. Randomized clinical trial comparing laparoscopic hiatal hernia repair using sutures versus sutures reinforced with non-absorbable mesh. Surg Endosc. 2018;32:4579-89.
6 Oelschlager BK, Pellegrini CA, Hunter JG, Brunt ML, Soper NJ, Sheppard BC, et al. Biologic prosthesis to prevent recurrence after laparoscopic paraesophageal hernia repair: long-term follow-up from a multicenter, prospective, randomized trial. J Am Coll Surg. 2011;213:461-8.

7 Memon MA, Memon B, Yunus RM, Khan S. Suture cruroplasty versus prosthetic hiatal herniorrhaphy for large hiatal hernia: a metaanalysis and systematic review of randomized controlled trials. Ann Surg. 2016;263:258-66.

8 Watson DI, Thompson SK, Devitt PG, Smith L, Woods SD, Aly A, et al. Laparoscopic repair of very large hiatus hernia with sutures versus absorbable mesh versus nonabsorbable mesh: a randomized controlled trial. Ann Surg. 2015;261:282-9.

9 Li J, Cheng T. Mesh erosion after hiatal hernia repair: the tip of the iceberg? Hernia. 2019;23: 1243-52.

10 Granderath FA, Schweiger UM, Pointner R. Laparoscopic antireflux surgery: tailoring the hiatal closure to the size of hiatal surface area. Surg Endosc. 2007;21:542-8.
11 von Elm E, Altman DG, Egger M, Pocock SJ, Gøtzsche PC, Vandenbroucke JP, et al. The strengthening the reporting of observational studies in epidemiology (STROBE) statement: guidelines for reporting observational studies. Int J Surg. 2014;12:1495-9.

12 Agha R, Abdall-Razak A, Crossley E, Dowlut $\mathrm{N}$, Iosifidis C, Mathew G, et al. STROCSS 2019 guideline: strengthening the reporting of cohort studies in surgery. Int J Surg. 2019; 72:156-65.

13 Clavien PA, Barkun J, de Oliveira ML, Vauthey JN, Dindo D, Schulick RD, et al. The Clavien-Dindo classification of surgical complications: five-year experience. Ann Surg. 2009; 250:187-96.

14 Schlosser KA, Maloney SR, Prasad T, Augenstein VA, Heniford BT, Colavita PD. Mesh reinforcement of paraesophageal hernia repair: trends and outcomes from a national database. Surgery. 2019;166:879-85.

15 Campos V, Palacio DS, Glina F, Tustumi F, Bernardo WM, Sousa AV. Laparoscopic treatment of giant hiatal hernia with or without mesh reinforcement: a systematic review and meta-analysis. Int J Surg. 2020;77:97-104. 
16 Watson DI, Thompson SK, Devitt PG, Aly A, Irvine $\mathrm{T}$, Woods SD, et al. Five year follow-up of a randomized controlled trial of laparoscopic repair of very large hiatus hernia with sutures versus absorbable versus nonabsorbable mesh. Ann Surg. 2020;272:241-7.
17 Targarona EM, Bendahan G, Balague C, Garriga J, Trias $\mathrm{M}$. Mesh in the hiatus: a controversial issue. Arch Surg. 2004;139:1286-96; discussion 1296.

18 Ilyashenko VV, Grubnyk VV, Grubnik VV. Laparoscopic management of large hiatal hernia: mesh method with the use of ProGrip mesh versus standard crural repair. Surg Endosc. 2018;32:3592-8.
19 Dallemagne B, Weerts J, Markiewicz S, Dewandre JM, Wahlen C, Monami B, et al. Clinical results of laparoscopic fundoplication at ten years after surgery. Surg Endosc. 2006;20: $159-65$. 